\title{
OPTIC DISC HEIGHT MEASUREMENT WITH THE ZEISS 4-MIRROR CONTACT LENS AND 78 DIOPTRE LENS COMPARED
}

\author{
A. F. SPENCER and S. A. VERNON \\ Nottingham
}

\begin{abstract}
SUMMARY
Measurements of optic disc diameter with the Zeiss 4mirror contact lens and 78 dioptre (D) lens, made by projecting a slit-beam of known height onto the image of the disc, were compared with planimetric measurements in 30 eyes. The $78 \mathrm{D}$ lens measurements were significantly larger than both the Zeiss lens and planimetric measurements $(p<0.0001$ and $p=0.0047$ respectively). The measurements using the Zeiss lens and planimetry did not differ significantly. Compared with planimetry, for the 24 eyes within 3 D of emmetropia, the correlation was greater for the Zeiss $(r=0.8591)$ than for the $78 \mathrm{D}$ lens $(r=0.7148)$. The Zeiss lens also showed better agreement and less scatter of results compared with the $78 \mathrm{D}$ lens (standard error of the mean $\pm 0.0373 \mathrm{~mm}$ for the Zeiss and $\pm 0.0418 \mathrm{~mm}$ for the $78 \mathrm{D}$ lens). The Zeiss contact lens measurements of optic disc diameter show stronger correlation and better agreement with planimetry than measurements using a $78 \mathrm{D}$ lens.
\end{abstract}

Optic disc size varies considerably among individuals. $^{1-3}$ Large optic discs are more likely to be classified as glaucomatous and small discs as normal on clinical examination. ${ }^{4}$ Therefore a simple clinical method for measuring optic disc size would be of value in assessing the glaucoma suspect. In addition, establishing that a disc is small may aid in the diagnosis of non-arteritic anterior ischaemic optic neuropathy, ${ }^{5}$ optic nerve head drusen ${ }^{6}$ and optic disc hypoplasia. ${ }^{7}$ Large optic discs have been reported in patients with normal tension glaucoma ${ }^{8}$ and in different racial groups. ${ }^{9}$

Established methods of measurement include planimetry, ${ }^{1,2,10,11}$ image analysis from stereophotography ${ }^{12-14}$ and confocal scanning laser ophthalmo-

Correspondence to: Miss A. F. Spencer, FRCS, FRCOphth, Department of Ophthalmology, University Hospital, Queen's Medical Centre, Nottingham NG7 2UH, UK. scopy. ${ }^{15}$ Such methods require either expensive specialist equipment or analysis after the patient has left the department. The spot size of a certain direct ophthalmoscope has recently been advocated by Gross and Drance ${ }^{16}$ for estimating optic disc size. However, this has not been quantified and can offer only a very rough approximation of disc size.

We have recently described the use of the Zeiss 4mirror contact lens at the slit-lamp biomicroscope to measure vertical optic disc diameter. ${ }^{17}$ The slit-beam of the biomicroscope is used to measure the virtual, erect image of the optic disc. The 90 dioptre (D) lens has been used in a similar manner, at the slit-lamp, to measure disc size, ${ }^{18}$ although it utilises a different optical principle, that of indirect ophthalmoscopy. The $78 \mathrm{D}$ lens yields a larger image size than the 90 $\mathrm{D}$ lens and may therefore be preferable.

Optic disc size can be assessed from measurements of vertical disc diameter. The area of the disc is proportional to the diameter squared. This relationship is not strictly linear due to the eccentricity of the disc, but it is apparent that a small difference in disc diameter will produce a more significant difference in disc area. The previous study with the Zeiss 4-mirror lens ${ }^{17}$ demonstrated that vertical disc diameter measurements equated well with planimetric measurements. The size of the discs measured ranged from 1.3 to $2.0 \mathrm{~mm}$ (mean $1.7 \mathrm{~mm}$ ). These results are consistent with other studies of disc size measured by planimetry. ${ }^{1,11}$ This provides a simple reference range for the clinician.

The aim of this study was to compare measurements of optic disc diameter obtained with the Zeiss 4-mirror contact lens, the $78 \mathrm{D}$ lens and established planimetric techniques. ${ }^{19}$

\section{METHODS}

Thirty patients were examined and one eye of each patient was included in the study. There were 10 
normals, 6 ocular hypertensives and 14 glaucoma patients. All the patients had a visual acuity of $6 / 9$ or better in the study eye and clear ocular media. Twenty-four eyes had a refractive error of up to \pm 3 $\mathrm{D}$ and 6 eyes between \pm 3 and $7 \mathrm{D}$.

Corneal curvature of the eyes was measured by a Javal Schiotz keratometer and spectacle refractions were performed by experienced optometric staff at a separate examination.

Informed consent was obtained after an explanation of the nature of the study, which followed the tenets of the Helsinki Declaration.

Vertical disc diameter was calculated using the formulae published by Bengtsson and Krakau. ${ }^{19}$ Photographs of the optic discs were taken at the 30 degree setting, the highest magnification, with a Topcon fundus camera. Following Bengtsson and Krakau's ${ }^{19}$ method a camera constant for the Topcon camera was calculated. The photographic slides of the optic discs were then projected onto a screen and the vertical diameter of the optic disc was measured using a scientific metal ruler. The same ruler was used by two independent observers (A and $\mathrm{B}$ ). The optic disc was defined as the area inside the white peripapillary scleral ring of Elschnig. The vertical diameter was defined as the distance from the edge of the nerve fibre rim at 12 o'clock to the edge of the nerve fibre rim at 6 o'clock. The mean image height measured by the two observers was used. The actual size of the optic disc was then calculated from the third correction described by Bengtsson and Krakau. ${ }^{19}$ This uses spectacle refraction and keratometry. In our previous studies ${ }^{11,14}$ measuring vertical disc diameter the best correlation and agreement with clinical methods was demonstrated with this correction.

The pupil was dilated with $1 \%$ tropicamide and the eye anaesthetised with proxymetacaine $0.5 \%$ or amethocaine $1 \%$. The Zeiss 4-mirror gonioscope contact lens was then applied gently to the cornea, the fundal image being observed through the central viewing zone. The optic disc was defined as above. A narrow vertical slit-beam of light was reduced progressively in size from $5 \mathrm{~mm}$ until it was judged to correspond to the size of the disc. The beam height was then recorded, from the scale on the slit-lamp, by an assistant. The slit-beam was then reset to $5 \mathrm{~mm}$ and the measurement repeated twice. The observer performing the measurements was thus unaware of the results until all three readings had been taken. As the height scale of the slit-lamp beam is calibrated in $0.1 \mathrm{~mm}$ steps, measurements were judged by the assistant to the nearest $0.1 \mathrm{~mm}, 0.05 \mathrm{~mm}$ increments being 'rounded up'. From the three readings a mean was derived.

On a separate occasion the same eyes were measured using the $78 \mathrm{D}$ condensing lens at the same slit-lamp biomicroscope by the same observer (S.A.V.). The pupil was dilated with g. tropicamide $1 \%$ and the vertical optic disc diameter measured by comparison with the slit-beam in an identical manner to the Zeiss lens measurements. Again the mean of the three measurements was calculated. The $78 \mathrm{D}$ lens used was manufactured by Volk. The lens has a magnification constant of 0.86 (instruction manual, Volk).

Prior to the study the slit-beam of the biomicroscope had been calibrated. ${ }^{19}$

\section{RESULTS}

The measurements using the $78 \mathrm{D}$ lens and the Zeiss 4-mirror contact lens are compared with the planimetric measurement, using Bengtsson and Krakau's third correction, in Figs. 1 and 2. The line of identity is plotted. The $78 \mathrm{D}$ lens measurements as plotted have not been corrected by the magnification factor for the lens. The Zeiss lens measurements ranged from 1.30 to $2.00 \mathrm{~mm}$ (mean $1.66 \mathrm{~mm}$ ) and the $78 \mathrm{D}$ lens measurements from 1.28 to $2.20 \mathrm{~mm}$ (mean $1.82 \mathrm{~mm})$.

The $78 \mathrm{D}$ lens measurements are seen to be larger than the planimetric and Zeiss lens measurements. If the magnification factor is not applied to the $78 \mathrm{D}$ lens measurements (as in Fig. 2) the results are, on average, $0.16 \mathrm{~mm}$ larger than those obtained with the Zeiss contact lens. The magnification correction increases this difference to $0.43 \mathrm{~mm}$. If the two methods of measurements are compared, for all 30 eyes, using the paired two-tailed Student's $t$-test, they are significantly different $(p<0.0001)$. The $78 \mathrm{D}$ lens measurements are also significantly different from the planimetric measurements by the same test $(p=0.0047)$. There is no significant difference between the Zeiss lens and planimetric measurements by the paired two-tailed Student's $t$-test $(p=0.1130)$.

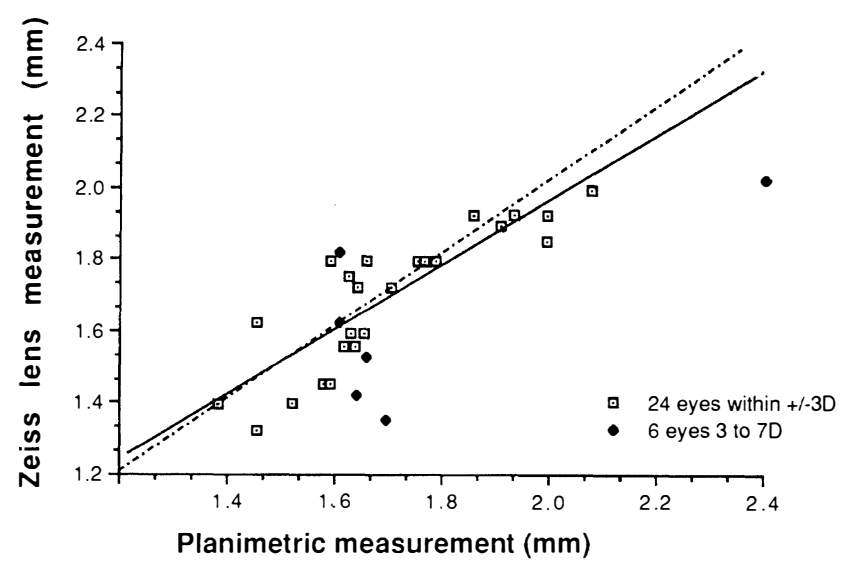

Fig. 1. Zeiss contact lens and planimetric measurements compared. Regression line for the 24 eyes within \pm 3 D of emmetropia is shown; the broken line is the line of equality. 


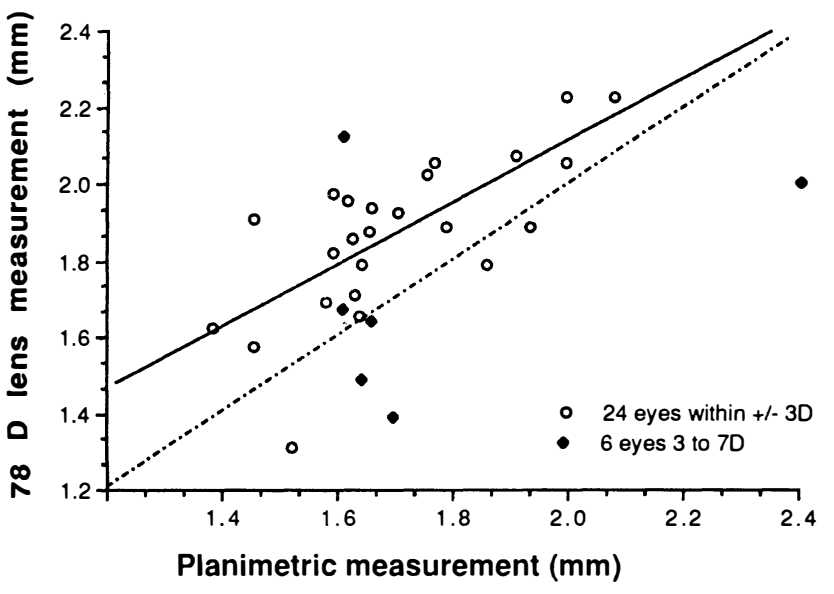

Fig. 2. The $78 \mathrm{D}$ lens (uncorrected measurements) and planimetric measurements compared. Regression line for the 24 eyes within $\pm 3 \mathrm{D}$ of emmetropia is shown; the broken line is the line of equality.

The correlation coefficients are detailed in Table I for both the clinical methods compared with the photographic measurements.

There is also a larger scatter in the $78 \mathrm{D}$ lens measurements (Fig. 1). For example, a disc measured as $1.6 \mathrm{~mm}$ by planimetry may measure $1.4-1.8 \mathrm{~mm}$ with the Zeiss lens but $1.35-2.1 \mathrm{~mm}$ with the $78 \mathrm{D}$ lens. The standard error of the mean is $\pm 0.0373 \mathrm{~mm}$ for the Zeiss lens and $\pm 0.0418 \mathrm{~mm}$ for the $78 \mathrm{D}$ lens. The standard error for the 'gold standard' of the planimetric measurement is similar to that for the Zeiss lens $( \pm 0.0385 \mathrm{~mm})$.

The Zeiss lens measurements and the indirect ophthalmoscopic measurements with the $78 \mathrm{D}$ lens can be compared directly. For 24 eyes the correlation coefficient $r=0.7348$ and for all 30 eyes $r=0.7874$.

Agreement with planimetry by a Bland and Altman plot $^{20}$ is demonstrated in Fig. 3 for the Zeiss lens (for 24 eyes within $\pm 3 \mathrm{D}$ of emmetropia). Similarly agreement is demonstrated between the $78 \mathrm{D}$ lens and planimetry in Fig. 4 (for 24 eyes). If the regression line is plotted for each of the graphs, it is not significant for either: $r=-0.105(p=0.617)$ for the Zeiss lens and $r=-0.177(p=0.398)$ for the $78 \mathrm{D}$ lens. Therefore neither method systematically over- or under-reads, as a function of the mean values, for a particular size of optic disc.

For the Zeiss lens $100 \%$ of the measurements of disc diameter are within $\pm 0.2 \mathrm{~mm}$, and $67 \%(16 / 24)$

Table I. Correlation coefficients for the $78 \mathrm{D}$ lens and Zeiss 4mirror contact lens compared with the third planimetric correction for the 24 eyes within $\pm 3 \mathrm{D}$ of emmetropia and for all 30 eyes

\begin{tabular}{lcc}
\hline Clinical method & 24 eyes & 30 eyes \\
\hline $78 \mathrm{D}$ lens & 0.7148 & 0.5495 \\
Zeiss contact lens & 0.8591 & 0.7714 \\
\hline
\end{tabular}

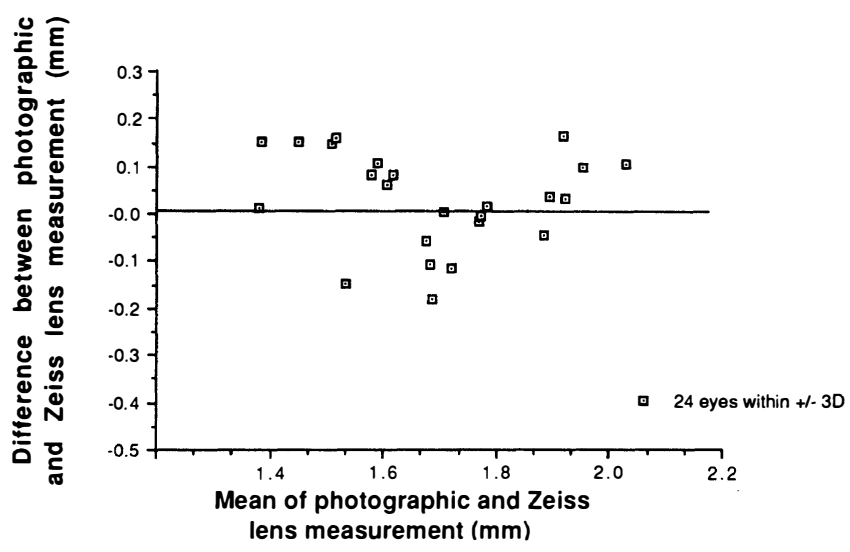

Fig. 3. Agreement between Zeiss contact lens and planimetric measurements. Continuous line is the line of equality.

within $\pm 0.1 \mathrm{~mm}$, of the mean difference between the planimetric and Zeiss lens measurements. For the $78 \mathrm{D}$ lens $83 \%(20 / 24)$ of the measurements are within $\pm 0.2 \mathrm{~mm}$ and $58 \%(14 / 24)$ are within \pm $0.1 \mathrm{~mm}$ of the mean difference between the planimetric and $78 \mathrm{D}$ lens measurements.

\section{Intra-observer Variation}

The coefficient of variation was calculated as the square root of the mean value of the variance of the measurements taken three times for each of the 30 optic discs and then divided by the mean value of the mean measured diameters.

For the Zeiss 4-mirror contact lens the mean variance was 0.0023 and the mean disc diameter $1.656 \mathrm{~mm}$. The coefficient of variation is therefore 0.029 or $2.9 \%$. For the $78 \mathrm{D}$ lens the mean variance was 0.0026 and the mean disc diameter $1.815 \mathrm{~mm}$. The coefficient of variation is therefore 0.028 or $2.8 \%$.

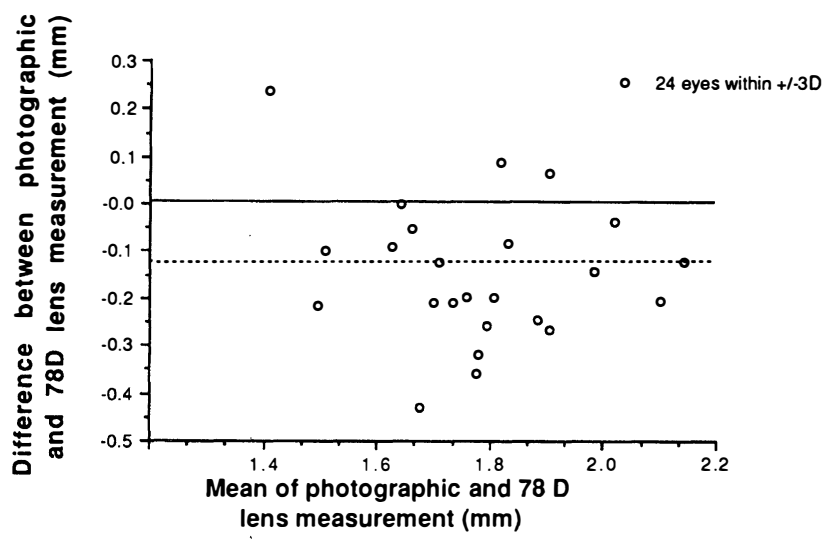

Fig. 4. Agreement between $78 \mathrm{D}$ lens and planimetric measurements. Continuous line is the line of equality; broken line is the mean difference between the measurements. 


\section{DISCUSSION}

Vertical disc diameter measurements made with the Zeiss 4-mirror contact lens at the slit-lamp biomicroscope correlate better with planimetric measurements than similar measurements made with the $78 \mathrm{D}$ lens. Our results differ from a recent publication by Ruben ${ }^{18}$ in which the $90 \mathrm{D}$ lens was used to estimate optic disc size, where a higher correlation with planimetric techniques was obtained. Ruben found the correlation between the $90 \mathrm{D}$ lens and planimetry to be $r=0.82$, which is similar to the stronger correlation found with the Zeiss lens in this study.

The $78 \mathrm{D}$ lens measurements are significantly larger than the planimetric measurements even when they have not been adjusted for the magnification factor of the lens. From these results it appears that the magnification factor of 0.86 quoted by the manufacturer is not correct. In addition the $78 \mathrm{D}$ lens results have much greater scatter than the Zeiss lens results when plotted against planimetry and when agreement is examined by a Bland and Altman plot. ${ }^{20}$ Also a substantial number of measurements lie outside $\pm 0.2 \mathrm{~mm}$ from the mean difference between the measurements. For example a disc measured as $1.6 \mathrm{~mm}$ by planimetry can be seen to measure between 1.4 and $1.8 \mathrm{~mm}$ using the Zeiss lens (Fig. 1). However, with the $78 \mathrm{D}$ lens this measurement may be between 1.35 and $2.10 \mathrm{~mm}$ (Fig. 2). Agreement cannot be compared with the $90 \mathrm{D}$ lens study $^{18}$ as this variable was not assessed by the author. If, however, the data from that study were to be re-plotted, it is likely that the $90 \mathrm{D}$ lens would overestimate the smaller optic discs. The $78 \mathrm{D}$ lens yields a larger image than the $90 \mathrm{D}$ lens and should improve the accuracy of measurements at the lower end of the scale, for it should be noted that the size of the slit-beam does not reduce below $1.0 \mathrm{~mm}$ on the linear scale of the Haag-Streit slit-lamp.

Assessment of the size of the optic disc, even by more sophisticated techniques, relies on the interpretation of the optic disc boundary. In this study both measurements were carried out by the same observer, who is experienced in optic disc assessment from previous studies. ${ }^{17,21}$ In addition the intraobserver variability for the two methods was similar. It would seem unlikely that the differences, either between the planimetric and clinical methods, or between the two clinical methods, were due to great variability between the observer's recognition of the optic disc boundary by the two methods.

Since early attempts to measure the optic disc, increasing refractive error has been known to cause inaccuracies in both direct and indirect ophthalmoscopic methods of measurement. ${ }^{22}$ Refractive error influences the image size produced, in indirect ophthalmoscopy, if the condensing lens does not coincide with the anterior focus of the eye. ${ }^{23}$ Montgomery, ${ }^{24}$ in his report of the Optic Disc Biometer, another indirect method of optic disc measurement, also emphasised the importance of the positioning of the condensing lens. At the slit-lamp biomicroscope with the $78 \mathrm{D}$ lens, the image obtained may appear 'focused' adequately to take a reading with the slit-beam when in fact the lens is not exactly at the anterior focus of the eye. This may account for some of the disparity between the readings obtained with the $78 \mathrm{D}$ lens and planimetry. However, the method itself is highly repeatable as seen by the low coefficient of variation for intraobserver variation.

Wells et al. $^{25}$ used an indirect ophthalmoscopic camera, with different power condensing lenses, to photograph the retina of enucleated eyes with solid domes (to mimic choroidal tumours) placed in the suprachoroidal space. The accuracy of the measurement of such 'fundus structures' from the photographs and the alterations associated with the different power and width of the condensing lenses and increasing axial length were calculated. The most significant finding was the alteration in the field of view and size of objects with increasing axial length. This suggests that the inaccuracies in our study are not merely produced by the position of the condensing lens but that a correction factor for the magnification of the eye may need to be calculated for eyes of greater refractive error. This is supported by the significantly greater disparity found between the measurements for the 6 eyes with refractive errors greater than $3 \mathrm{D}$.

The Zeiss 4-mirror contact lens also shows a poorer correlation for the eyes with greater refractive errors, but this is much less marked than for the $78 \mathrm{D}$ lens. The contact lens method may be more accurate because the lens is in contact with the eye and does not have to be held precisely at the anterior focus of the eye, i.e. the change in image size ${ }^{23,25}$ due to alteration of focus and field of view is avoided. This would be expected to reduce the error induced by increasing axial length. In addition in indirect ophthalmoscopy, decentration of the object from the visual axis increases the viewing angle and alters the relative magnification. Data from two studies ${ }^{25,26}$ suggest that there is an $11 \%$ decrease in field size when the equator is reached. If the optic disc was decentred when measured then this would introduce further errors. This is more likely with the $78 \mathrm{D}$ lens than with the Zeiss lens as the field of view with the Zeiss lens is smaller and it is not really possible to measure the disc with the slit-beam in a decentred position.

Littmann' ${ }^{27}$ calculations have shown that the individual ocular magnification factor depends mainly on the anterior corneal curvature, spectacle 
refraction and axial length, in that order. The posterior corneal curvature, anterior chamber depth, anterior and posterior lens curvature and lens thickness are much less important. Littmann had calculated that a modification of $20 \%$ in the corneal thickness, anterior chamber depth, lens thickness or the lens radii, resulted in a change in magnification of the eye of $0.07 \%, 0.6 \%, 0.5 \%$ and $4.6 \%$ respectively. In comparison a $20 \%$ variation of the corneal curvature results in a change in the ocular magnification of $14.6 \%$. Therefore by eliminating the main source of change in the magnification of the eye - the corneal curvature - the contact lens method should be more accurate than an indirect method of measurement.

In addition, using a contact lens removes the reflections from the anterior surface of the cornea and the posterior surface of the $78 \mathrm{D}$ lens ${ }^{28}$ and may therefore aid the observer in accurately determining the boundary of the optic disc.

Further differences in the comparison between the clinical and planimetric methods may be due to inaccuracies in the planimetric techniques. Bengtsson and Krakau's previous calculations have been shown to be equivalent to Littmann's algorithms to correct for the magnification factor of the eye, when using the Zeiss fundus camera. ${ }^{29}$ Other investigators have demonstrated that decentration of the object, alteration of the eye-to-camera distance and increasing ametropia can cause a large variation between the measured and calculated magnification..$^{30-32}$ However, a good correlation has been shown between the planimetric correction used and optic disc measurement with the Heidelberg Retina Tomograph confocal scanning laser ophthalmoscope. ${ }^{21}$

Both the Zeiss 4-mirror contact lens and the $78 \mathrm{D}$ lens techniques appear to be repeatable, with coefficients of variation of $2.9 \%$ and $2.8 \%$ respectively. This is comparable to the $3.07 \%$ found by Ruben in his study using the $90 \mathrm{D}$ lens. ${ }^{18}$

Ruben suggested that a correction for the $90 \mathrm{D}$ lens could be calculated from the regression line for the graph and then applied to the $90 \mathrm{D}$ lens measurements. Theoretically a similar correction could be calculated for the $78 \mathrm{D}$ lens. However, the linear magnification of the condensing lens varies considerably even in ametropia and this is therefore an oversimplification which may underestimate the size of the optic disc considerably. ${ }^{33}$ The $78 \mathrm{D}$ lens may be a useful technique for a rapid assessment of whether the optic disc is large or small, and provided the clinician knows the normal range of disc size for this method, the results from this study can be taken as a guide. However, the measurement obtained cannot be considered to be an accurate assessment of optic disc size. From this analysis it appears that measurement of the optic disc diameter with the
Zeiss 4-mirror contact-lens at the biomicroscope is in closer agreement with optic disc diameter calculated using planimetric corrections.

\section{REFERENCES}

1. Jonas JB, Gusek GC, Guggenmoos-Holzmann I, Naumann GOH. Variability of the real dimensions of normal human optic discs. Graefes Arch Clin Exp Ophthalmol 1988;226:332-6.

2. Bengtsson $B$. The variation and covariation of cup and disc diameters. Acta Ophthalmol (Copenh) 1976; 54:804-18.

3. Quigley HA, Brown AE, Morrison JD, Drance SM. The size and shape of the optic disc in normal human eyes. Arch Ophthalmol 1990;108:51-7.

4. Heijl A, Molder H. Optic disc diameter influences the ability to detect glaucomatous disc damage. Acta Ophthalmol (Copenh) 1993;71:122-9.

5. Beck RW, Savino PJ, Repka MX, et al. Optic disc structure in anterior ischaemic optic neuropathy. Ophthalmology 1984;91:1334-7.

6. Spencer WH. Drusen of the optic disc and aberrant axoplasmic transport. The XXXIV Edward Jackson Memorial Lecture. Am J Ophthalmol 1978;85:1-12.

7. Beuchat L, Safran AB. Optic nerve hypoplasia: papillary diameter and clinical correlation. J Clin Neuro-ophthalmol 1985;5:249-53.

8. Burk ROW, Rohrschneider K, Noack H, Volcker HE. Are large optic nerve heads susceptible to glaucomatous damage at normal intraocular pressure? A threedimensional study by scanning laser tomography. Graefes Arch Clin Exp Ophthalmol 1992;230:552-60.

9. Chi T, Ritch R, Stickler D, Pitman B, Tsai C, Hsieh FY. Racial differences in optic nerve head parameters. Arch Ophthalmol 1989;107:836-9.

10. Balazsi AG, Drance SM, Schulzer M, Douglas GR. Neuroretinal rim area in suspected glaucoma and early chronic open-angle glaucoma. Arch Ophthalmol 1984;102:1011-4.

11. Jonas JB, Gusek GC, Naumann GOH. Optic disc, cup and neuroretinal rim size, configuration and correlations in normal eyes. Invest Ophthalmol Vis Sci 1988;29:1151-8.

12. Takamoto T, Schwartz B. Reproducibility of photogrammetric optic disc cup measurements. Invest Ophthalmol Vis Sci 1985;26:814-7.

13. Schwartz B. New techniques for the examination of the optic disc and their clinical application. Trans Am Acad Ophthalmol Otolaryngol 1976;81:227-5.

14. Portney GL. Photogrammetric analysis of volume asymmetry of the optic nerve head cup in normal, hypertensive and glaucomatous eyes. Am J Ophthalmol 1975;80:51-5.

15. Cioffi GA, Robin AL, Eastman RD, Perell HF, Sarfarazi FA, Kelman SE. Confocal laser scanning ophthalmoscope: reproducibility of optic nerve head topographic measurements with the confocal laser scanning ophthalmoscope. Ophthalmology 1993; 100:57-62.

16. Gross PG, Drance SM. Comparison of a simple ophthalmoscopic and planimetric measurement of glaucomatous neuroretinal rim areas. J Glaucoma 1995;4:314-6:

17. Spencer AF, Vernon SA. Optic disc measurement with the Zeiss 4-mirror contact-lens. $\mathrm{Br} \mathrm{J}$ Ophthalmol 1994;78:775-80.

18. Ruben S. Estimation of optic disc size using indirect biomicroscopy. Br J Ophthalmol 1994;78:363-4. 
19. Bengtsson B, Krakau CET. Correction of optic disc measurements on fundus photographs. Graefes Arch Clin Exp Ophthalmol 1991;230:24-8.

20. Bland JM, Altman DG. Statistical methods for assessing agreement between two methods of clinical measurement. Lancet 1986;1:307-10.

21. Spencer AF, Vernon SA. Vertical optic disc diameter: the Heidelberg Retina Tomograph versus photographs. Invest Ophthalmol Vis Sci 1995;36:796-803.

22. Franceschetti A, Bock RH. Megalopapilla: a new congenital anomaly. Am J Ophthalmol 1950;33:227-34.

23. Elkington A, Frank H. Clinical optics. Oxford: Blackwell Scientific, $1984: 134$.

24. Montgomery DMI. The optical spacer: a simple device which extends the scope of indirect ophthalmoscopy. Br J Ophthalmol 1992;76:45-6.

25. Wells E, Barrall J, Martin D. Fundus measurements with indirect ophthalmoscopy: an experimental approach. Arch Ophthalmol 1992;110:1303-8.

26. Enoch J, Goldberg M. Lateral and longitudinal magnification in direct and indirect ophthalmoscopy. Arch Ophthalmol 1971;86:536-47.
27. Littmann $H$. The determination of the true size of objects in the background of the living eye. Klin Monatsbl Augenheilkd 1982;180:286-9.

28. Colenbrander A. Principles of ophthalmoscopy. In: Duane TD, Jaeger AE, editors. Clinical ophthalmology, vol 1. Philadelphia: JB Lippincott, 1989:chap 63-19.

29. Mansour AM. Measuring fundus landmarks. Invest Ophthalmol Vis Sci 1990;31:41-2.

30. Pach J, Pennell DO, Romano PE. Optic disc photogrammetry: magnification factors for eye position, centration, and ametropias, refractive and axial; and their application in the diagnosis of optic nerve hypoplasia. Ann Ophthalmol 1989;21:454-62.

31. Lotmar W. Dependence of magnification upon the camera-to-eye distance in the Zeiss fundus camera. Acta Ophthalmol (Copenh) 1984;62:131-4.

32. Arnold JV, Gates JWC, Taylor KM. Possible errors in the measurement of retinal lesions. Invest Ophthalmol Vis Sci 1993;34:2576-80.

33. Barr DB. Estimation of optic disc size. Br J Ophthalmol 1995;79:298. 